\title{
Eine habsburgische Quelle im Kampf gegen Frankreich: Vollmacht Kaiser Friedrichs III. an König Maximilian, 1493
}

\author{
Marina Blum \\ Kerngebiet: Mittelalter \\ eingereicht bei: Mag. Dr. Gertraud Zeindl \\ eingereicht im: SoSe 2020 \\ Rubrik: Proseminar-Arbeit
}

\begin{abstract}
A Habsburg Source in the Struggle against France: The Power of Attorney of Emperor Frederick III to King Maximilian, 1493
\end{abstract}

This paper deals with a medieval source that has received little attention in research so far: the power of attorney of Emperor Frederick III to King Maximilian of 1493. This historical document, which calls for the punishment of the French king, is marked by years of conflict between Maximilian I and Charles VIII of France. The source provides a crucial insight into the propaganda of Frederick III and Maximilian I.

\section{Einleitung}

Diese Proseminararbeit betrachtet eine Quelle, die nicht nur an einem Wendepunkt der europäischen Geschichte, am Übergang vom Mittelalter zur Frühen Neuzeit, sondern auch in einem Umbruchsjahr für das Haus Habsburg entstand: die Vollmacht Kaiser Friedrichs III. an seinen Sohn König Maximilian vom Februar 1493. Nur wenige Monate später übernahm Maximilian I. nach dem Tod seines Vaters die Alleinherrschaft über das Heilige Römische Reich.' In diesem Zusammenhang wird folgender Fragestellung nachgegangen: Warum kam es zur Entstehung und Vervielfältigung der Vollmacht von Kaiser Friedrich III. an König Maximilian im Februar 1493 mit dem Aufruf zur Bestrafung des französischen Königs? Für die Diskussion dieser Fragestellung wird in einem ersten

Hermann Wiesflecker, Kaiser Maximilian I. Das Reich, Österreich und Europa an der Wende zur Neuzeit, Bd. 1 : Jugend, burgundisches Erbe und Römisches Königtum bis zur Alleinherrschaft. 1459-1493, Wien-München 1971, S. $351,355,406$ 
Schritt die Quelle fokussiert. Im Rahmen einer Quellenbeschreibung werden die äuBeren und inneren Merkmale der Vollmacht vorgestellt, danach knüpft eine Quelleninterpretation an, die den beiden Teilaspekten des Dokuments und der Fragestellung nachgeht: (a) dem zerrütteten Verhältnis zwischen Maximilian I. und König Karl VIII. von Frankreich; (b) der Vervielfältigungsart durch Inkunabeln bzw. der Funktion dieser Vollmacht. Aus der Verbindung von innerer und äußerer Quellenkritik ergibt sich folgende These für die Interpretation: Die jahrelangen Auseinandersetzungen zwischen MaximiIian I. und Karl VIII., aber auch propagandistische Gründe führten zur Ausstellung und Verbreitung der Vollmacht. Schließlich werden in einem Fazit die Erkenntnisse zusammengefasst.

Wie die vorgestellte Gliederung zeigt, wurde für diese Proseminararbeit als Methodik eine Kombination aus Quellenerschließung und Literaturarbeit gewählt. Vor allem für die Interpretation der Quelle wurden verschiedenste Sekundärwerke herangezogen. Hierzu ein Überblick: In Hinblick auf die Konflikte zwischen Maximilian I. und Karl VIII. stützt sich die Proseminararbeit besonders auf Ausführungen von Christina Lutter ${ }^{2}$ und Susanne Wolf3. Während erstere ihren Fokus auf den Sohn Maximilian legt, finden sich bei Wolf die verwobenen Herrscherhandlungen der beiden Doppelregenten wieder. Für das letzte Jahr der Konfrontation wurde eine Publikation von Hermann Wiesflecker ${ }^{4}$ herangezogen, für die Literaturarbeit zur Funktion der Vervielfältigung durch den Druck der Vollmacht unter anderem Aufsätze von Jan-Dirk Müller ${ }^{5}$, Reinhard Seyboth ${ }^{6}$ und Manfred Hollegger?.

Wie die Titel der Sekundärwerke zeigen, gibt es Forschungsliteratur zur Auseinandersetzung zwischen Maximilian I. und Karl VIII. sowie zur Propaganda bzw. Publizistik von Maximilian I. Speziell zur vorliegenden Quelle sind jedoch kaum spezifische Forschungen vorhanden. Daraus ergibt sich die Relevanz dieser Proseminararbeit.

\section{Quellenbeschreibung}

Bei der vorliegenden Vollmacht von Kaiser Friedrich III. an König Maximilian vom Februar 1493 handelt es sich um eine Textquelle. Im Konkreten ist sie bei den Quellenarten

2 Christina Lutter, Maximilian I. (1486-1519), in: Bernd Schneidmüller/Stefan Weinfurter (Hrsg.), Die Deutschen Herrscher des Mittelalters. Historische Portraits von Heinrich I. bis Maximilian I. (919-1519), München 2003, S. 518-542.

3 Susanne Wolf, Die Doppelregierung Kaiser Friedrichs III. und König Maximilians (1486-1493) (Forschungen zur Kaiser- und Papstgeschichte des Mittelalters. Beihefte zu J. F. Böhmer, Regesta Imperii 25), Köln-Mainz 2005.

$4 \quad$ Wiesflecker, Kaiser Maximilian I.

5 Jan-Dirk Müller, Publizistik unter Maximilian I. Zwischen Buchdruck und mündlicher Verkündigung, in: Ute Frevert/ Wolfgang Braungart (Hrsg.), Sprachen des Politischen. Medien und Medialität in der Geschichte, Göttingen 2004, S. 95-122.

6 Reinhard Seyboth, Die Auseinandersetzung König Maximilians I. mit König Karl VIII. von Frankreich um die Bretagne im Spiegel zeitgenössischer Medien, in: Maximilian Lanzinner/Arno Strohmeyer (Hrsg.), Der Reichstag 1486-1613. Kommunikation - Wahrnehmung - Öffentlichkeiten (Schriftenreihe der Historischen Kommission bei der Bayerischen Akademie der Wissenschaften 73), Göttingen 2006, S. 239-257.

7 Manfred Hollegger, Erwachen und aufsten als ein starcker stryter. Zu Formen und Inhalt der Propaganda Maximilians I., in: Karel Hruza (Hrsg.), Propaganda, Kommunikation und Öffentlichkeit (11.-16. Jahrhundert) (Forschungen zur Geschichte des Mittelalters 6), Wien 2002, S. 223-234. 
als Urkunde in Form einer Inkunabel einzuordnen. Der Text enthält also Inhalts- und Strukturmerkmale einer Urkunde (siehe Kapitel "Innere Quellenkritik“), mit Blick auf die technische Herstellung liegt aber gleichzeitig eine Inkunabel vor. Unter Inkunabeln sind frühe Drucke zu verstehen, die noch vor 1501 entstanden und aufgrund der lateinischen Wortwurzel (lat. incunabula für dt. Windeln, Wiege) auch unter dem Terminus „Wiegendruck" bekannt sind. ${ }^{8}$

Beim Beschreibstoff der Quelle handelt es sich um Papier und der Text wurde in Hochformat aufgedruckt. Die Urkunde wurde in der Mitte gefaltet und weist eine gotische Buchschrift und Minuskel auf. Außerdem enthält der Einblattdruck mittig und mit einigem Abstand zum Text im unteren Drittel der Seite ein Wasserzeichen. Auf der Rückseite sind rote Wachsreste von einem aufgedrückten Siegel zurückgeblieben. ${ }^{9}$ Der Erhaltungszustand der Quelle ist relativ gut. Abgesehen von einigen Falten und Knicken an den Rädern bzw. von der Faltung zum Brief weist sie keine physischen Zerstörungen auf. $^{10}$

Die untersuchte Quelle wird im Tiroler Landesarchiv aufbewahrt. ${ }^{11}$ Der Quellentext dieser Urkunde findet sich darüber hinaus in den Regesta Imperii wieder und außerdem beschäftigen sich eine unveröffentlichte Edition des Tiroler Landesarchives sowie die in der Einleitung erwähnten Publikationen von Wiesflecker und Seyboth mit ihr. ${ }^{12}$

\section{$2.1 \quad$ Äußere Quellenkritik}

Wie der Quelle entnommen werden kann, wurde die Urkunde in Linz am 11. Februar 1493 nach der Inkarnationsära (also nach der Geburt Christi) ausgestellt. Angegeben wird die Datierung der Quelle aber auch in Anlehnung an die Zählung nach den Regierungsjahren des Ausstellers, Kaiser Friedrichs III.: im 53. Regierungsjahr als König des Heiligen Römischen Reiches, im 41. Regierungsjahr als Kaiser des Reiches und im 34. Regierungsjahr als König von Ungarn. Empfänger der Vollmacht war sein Sohn Maximilian. ${ }^{13}$

Da diese Vollmacht in Form einer Inkunabel vorliegt, muss auch ihren Entstehungsumständen nachgegangen werden. Die Entstehungszeit des Druckes ist nicht fassbar und auch der Drucker sowie der Ort sind unklar. Allerdings gibt das Österreichische Staatsarchiv, dem ebenfalls eine Inkunabel mit diesem Inhalt vorliegt, zwei Möglichkeiten an: den Druck bei Michael Furter in Basel oder bei Kilian Fischer in Freiburg/Breisgau. ${ }^{14}$ Die

8 Klaus Gantert, Handschriften, Inkunabeln, Alte Drucke. Informationsressourcen zu historischen Bibliotheksbeständen (Bibliotheks- und Informationspraxis 60), Berlin-Boston 2019, S. 133.

9 Vollmacht Kaiser Friedrichs III. an König Maximilian, 1493. TLA, Inkunabel 11, 1493 II 11; TLA, Quellenedition zu Inkunabel 11, Innsbruck 2019 (unveröffentlichtes Manuskript). Kopie im Besitz der Verfasserin.

10 Vollmacht Kaiser Friedrichs III. an König Maximilian, 1493. TLA, Inkunabel 11, 1493 II 11.

11 Ebd.

12 Paul-Joachim Heinig (Bearb.), Die Urkunden und Briefe aus dem Stadtarchiv Frankfurt am Main (Regesten Kaiser Friedrichs III. (1440-1493). Nach Archiven und Bibliotheken geordnet 4), Wien-Köln-Graz 1986, http://www. regesta-imperii.de/regesten/13-4-0-friedrich-iii/nr/1493-02-11_1_0_13_4_0_10623_1046, eingesehen 19.2.2021; TLA, Quellenedition; Wiesflecker, Kaiser Maximilian I.; Seyboth, Auseinandersetzung.

13 Vollmacht Kaiser Friedrichs III. an König Maximilian, 1493. TLA, Inkunabel 11, 1493 II 11.

14 Vollmacht Kaiser Friedrichs III. an König Maximilian, 1493. AT-OeStA/HHStA, SB Einblattdrucke 5, https://www. archivinformationssystem.at/detail.aspx?ID=288086, eingesehen 19.2.2021. 
weiteren Empfänger der Inkunabel sind ebenfalls nicht identifizierbar, da jedoch diese technische Vervielfältigung des Druckes gewählt wurde, ist eine breitere Empfängergruppe anzunehmen. ${ }^{15}$

Zur Echtheit der Quelle lässt sich Folgendes sagen: Da es sich bei der Quelle um eine Inkunabel handelt, liegt der ursprüngliche Text nicht als Original vor - den Regesta Imperii zufolge ist jenes bisher nicht auffindbar ${ }^{16}$-, sondern wurde technisch aufbereitet. Die Siegelreste und das Wasserzeichen sprechen jedoch für die Echtheit.

\subsection{Innere Quellenkritik}

Die zentralen Personen dieser Textquelle sind Kaiser Friedrich III. und sein Sohn Maximilian. Erwähnung findet in der Quelle aber auch der französische König Karl VIII. Sprachduktus und Wortwahl der Vollmacht entsprechen der Quellenart der Königs- bzw. Kaiserurkunde. Das gilt auch für den Aufbau entlang der klassischen Urkunden-Elemente: Protokoll - Kontext - Eschatokoll ${ }^{17}$.

Das Protokoll beginnt zwar nicht mit einer Anrufung Gottes (Invocatio), dafür jedoch mit dem Titel des Ausstellers (Intitulatio) samt Devotionsformel und daher mit der Legitimierung durch Gott ${ }^{18}$ :II ${ }^{19}$ Friderich von gots gnaden Rômischer keyser zůallenzeitten merer des Reichs / zů Hungern / Dalmatien / Croatien etc. Kunig / Hertzog zů ôsterreich / zů Steir / zů Kernndten / vnd zů Crain / Graue zů Tyrol etc. "20 Diese Titel verraten, dass Friedrich III. zum Ausstellungsdatum der Quelle die Herrschaft über Ungarn, Dalmatien, Kroatien, Österreich, Steiermark, Kärnten, Krain und Tirol innehatte.

Danach folgt schon der Übergang in den Hauptteil der Quelle, den Kontext: „Bekennen offennlich mit disem brief vnd tůn kunt al/lermeniglich [...] vnd geben auch wissentlich in crafft diss briefs." ${ }^{21}$ Die Publicatio (Promulgatio), die Verkündigungsformel, knüpft nämlich direkt an die Auflistung der Titel an. ${ }^{22}$ Der eigentliche Rechtsinhalt (Dispositio) ${ }^{23}$ dieser Kundmachung wird dann auf den Punkt gebracht, also die vollkommene Machtund Gewaltübertragung an den Sohn, König Maximilian I. (seine Titel werden ebenfalls angeführt), sowie der Befehl an diesen gegen den französischen König aufgrund seiner Handlungen gegen das Reich und Maximilian durch ein Aufgebot aller Lehensleute vorzugehen. ${ }^{24}$ Auch eine Strafandrohung bzw. Pönformel (Sanctio) ${ }^{25}$ kommt vor: „Ob sich auch Ir einer oder mer / derselben hilf sperren oder widern wûrde / die vmb solich pe/ne straffe vnd půsse durch seiner kûniclichen Camerprocurator Fiscal fûrnemen /

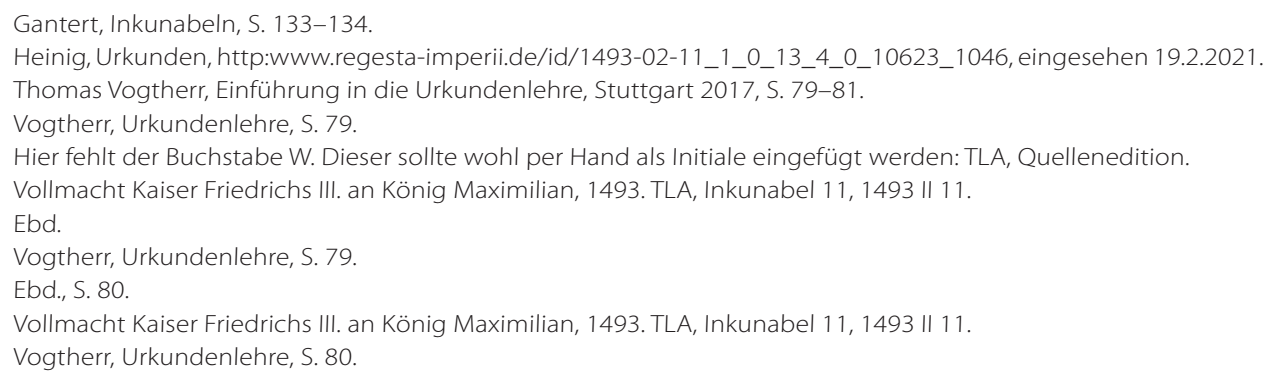


vnd die von Inen einbringen. "26 Maximilian I. wurde damit gewährt, gemeinsam mit dem Kammerprokurator-Fiskal gegen Ungehorsame vorzugehen. Abgeschlossen wird der Kontext mit den Worten "Mit vrkund diß briefs besigelt mit vnnserm keiserlichen anhanngundem Innsigel“27, also mit dem Verweis auf die Besiegelung (Corroboratio) der Quelle. ${ }^{28}$ Das Eschatokoll besteht aus einer Datumszeile ${ }^{29}$ :

„Geben zů Lynntz am Eilfften tag des monets February / Nach Cristi gepurt vierzehenhunndert vnd im drewundnewntzigisten / vnnser Reiche des Römischen im drewundfûnfftzigisten / Des keiserthumbs im Einunduiertzigisten vnd des hungerischen im vierunddreissigisten Jaren." ${ }^{130}$

Auf die unterschiedlichen Jahresangaben bzw. Zählweisen wurde im Kapitel „Äußere Quellenkritik" bereits eingegangen.

\section{Quelleninterpretation}

Als Ausgangspunkt für die Interpretation soll nochmal der Inhalt der Quelle auf den Punkt gebracht werden: Zusammenfassend geht es in der Inkunabel darum, dass Kaiser Friedrich III. seinem Sohn Maximilian I. eine Vollmacht für den Krieg gegen Frankreich ausstellt sowie darin das gesamte Reich zur Unterstützung aufruft. Die betroffenen geografischen Gebiete sind also das Heilige Römische Reich und Frankreich. ${ }^{31}$ Zeitlich lässt sich die Rechtsquelle am Ende des 15. Jahrhunderts, kurz vor dem Übergang der habsburgischen Doppelherrschaft zur Alleinherrschaft Maximilians, verorten. ${ }^{32}$ Im Rahmen der Quelleninterpretation geht es um das Warum der Beobachtungen, also um die Entstehungs- und Vervielfältigungsgründe der Quelle. Wie im Anschluss ausgeführt wird, sind dafür das Konfliktpotential zwischen Maximilian I. und Karl VIII. sowie Maximilians Medienkompetenz entscheidend. Aber auch die Position des Ausstellers, also des Kaisers, spielt eine Rolle.

\subsection{Zum zerrütteten Verhältnis zwischen Maximilian I. und Karl VIII.}

Für das Entstehen der Vollmacht mit der Aufrufung zur Bestrafung des französischen Königs ist es nicht mit dem Schildern eines historischen Ereignisses getan. Vielmehr war die Beziehung von Maximilian I. und Karl VIII. von jahrelangen, der Vollmacht vorausgehenden Auseinandersetzungen geprägt. Das folgende Unterkapitel unternimmt den Versuch, besagten vielschichtigen Konflikt zu skizzieren, wobei zwei Räume zentral sind: Burgund und die Bretagne..$^{33}$

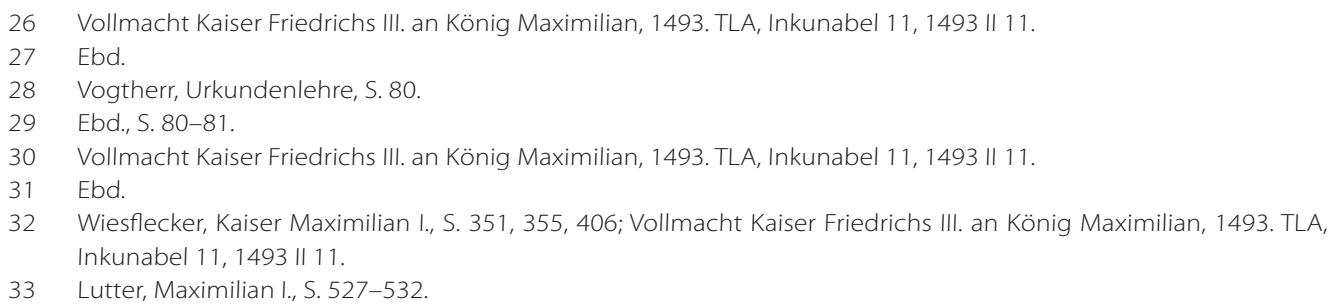




\subsubsection{Erbfolgekrieg in Burgund}

Die Auseinandersetzung der beiden Könige nahm schon vor ihrer Regentschaft ihren Anfang. Bereits in den 1470er-Jahren gab es Spannungen zwischen ihren Vätern - Kaiser Friedrich III. und König Ludwig XI. Mit dem Tod Karls des Kühnen, des Herzogs von Burgund, 1477 kam es zum Erbfolgekrieg. Beide Seiten erhoben Anspruch auf das Herzogtum, denn Burgund umfasste in der Zeit Karls des Kühnen sowohl Reichslehen als auch französische Lehen. Außerdem handelte es sich bei den burgundischen Herzögen um eine französische Nebenlinie. Der Bezug der Habsburger zu Burgund hatte sich aber ebenfalls auf verwandtschaftlicher Ebene verdichtet. Im Todesjahr Karls des Kühnen wurden nämlich die burgundische Herzogstochter, Maria von Burgund, und Maximilian vermählt, obwohl der französische König Ludwig XI. ähnliche Pläne für seinen Sohn hatte. ${ }^{34}$

Während des burgundischen Erbfolgekriegs, der eineinhalb Dekaden dauern sollte, kam es des Weiteren zu anderen hervorhebungswürdigen Ereignissen, die das Verhältnis Maximilians und König Karls VIII. prägten. Der unerwartete Tod von Maximilians Ehefrau Maria im Jahr 1482 verschärfte die Rivalitäten um Burgund. Maximilian besaß zwar persönlich kein erbrechtliches Anrecht auf das Herzogtum, wurde jedoch als Vormund der beiden gemeinsamen Kinder und Erben von Burgund als Gefahr wahrgenommen. Da Flandern die durch diese Entwicklungen erhaltenen Regentschaftsansprüche von Maximilian nicht anerkannte und sogar (militärische) Unterstützung aus Frankreich erhielt, erhöhte sich der Druck auf Maximilian. Im Dezember 1482 kam es daher zum Frieden von Arras. In diesem Vertrag wurde die Hochzeit zwischen der burgundischen Erbin, Maximilians Tochter Margarete und dem französischen Thronfolger, Karl VIII. rechtlich fixiert. Die Mitgift, die Gebiete Artois und Burgund, sowie Margarete selber gingen direkt an den französischen Hof über. ${ }^{35}$

\subsubsection{Gefangenschaft in Brügge}

Der mit dem Vertrag von Arras geschaffene Friede währte jedoch nicht lange. Der neue französische König Karl VIII. unterstütze, wie einst sein Vater (Tod Ludwigs XI. im August 1483), die starke und sich Maximilian widersetzende Grafschaft Flandern (Gent, Brügge, Ypern) und ließ Maximilian gegen Jahresende 1484 eine Kriegserklärung zukommen. ${ }^{36}$

„In den Jahren der nun folgenden Auseinandersetzungen eskalierte die Gewalt: Unter den zahlreichen traurigen Höhepunkten befinden sich [...] das blutige Strafgericht Maximilians in Gent (1485) ebenso wie seine eigene wochenlange Festsetzung in Brügge (1488).".37 
Letztere ergab sich als Folge aus der Rebellion Flanderns, vor allem Gents, im Jahr 1487, welche erneut Unterstützung durch Karl VIII. fand. Um Flandern vor französischen Angriffen zu schützen, forderte Maximilian von Brügge, wo er sich befand, militärische Hil$\mathrm{fe}$, die jedoch ausblieb. Auch seine herannahenden Truppen halfen ihm nicht, vielmehr verursachten sie Angst und Unruhe sowie das Schließen der Stadttore: Maximilian war gefangen. ${ }^{38}$

Diese Situation veranlasste den Kaiser zum Eingreifen: Das Reichsheer nahte zur Befreiung des Sohnes und Friedrich III. selbst führte es an. Als Grund nannte der Kaiser seine Vaterrolle ${ }^{39}$, vor allem aber die „Pflicht zur Verteidigung der Ehre des Reiches" ${ }^{40}$. Noch vor seiner Ankunft wurde Maximilian freigelassen, dennoch wollte der Kaiser diese Taten bestrafen. ${ }^{41}$ An dieser Stelle strebte er - anders als in der vorliegenden Quelle noch keinen Reichskrieg mit Frankreich an. ${ }^{42}$

Dennoch hatte die Gefangenschaft Maximilians bei den Aufständischen, die Karl VIII. eindeutig unterstützt hatten, langfristige Folgen. Von dieser abgelenkt bzw. beschäftigt konnte Maximilian Herzog Franz II. von Bretagne im Kampf gegen Frankreich nicht eigenhändig unterstützen. Die daraus resultierende Niederlage brachte den Friedensvertrag zwischen Frankreich und der Bretagne 1488 hervor, die dem französischen König viel Macht über das Herzogtum verlieh. ${ }^{43}$ Tatsächlich verstarb kurz darauf Franz II. von Bretagne und das Herzogtum musste sich „in d[ie] Händ[e] einer vormundschaftlichen Regierung"44 begeben.

\subsubsection{Bretonischer Ehekrieg}

Wie die letzten Ausführungen gezeigt haben, rückt zunehmend das erwähnte Stichwort "Bretagne" in den Vordergrund. Inhalt war der 1489 abgeschlossene Frankfurter Friedensvertrag zwischen Maximilian, seinem Sohn und Karl VIII., da die im Kampf gegen Franz II. errungenen Gebiete an die Bretagne zurückgehen sollten. In den meisten Vertragspunkten erkannte Maximilian große Nachteile. Seine Einwilligung ergab sich letztlich nur aufgrund des Drängens des Kaisers, da jener einen europäischen Frieden im Kampf gegen die Osmanen als notwendig erachtete. ${ }^{45}$

Erneut blieb der Friede mit Frankreich nicht von Dauer. Ende 1490 gab Maximilian „das Signal zur Rückkehr in den früheren Konfrontationskurs" ${ }^{\prime 4}$. Maximilian entschied sich für den politischen Schachzug, Anna von Bretagne, die Tochter des verstorbenen Herzogs Franz II., zu heiraten, was zumindest auf dem Papier durchgeführt wurde. ${ }^{47}$ In der

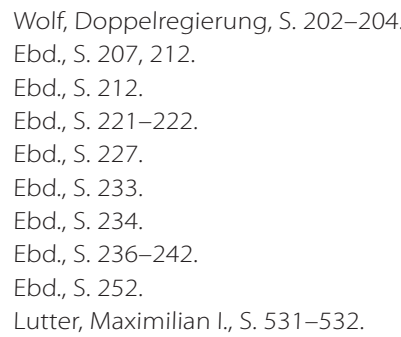


Folge wollte Maximilian rasch in die Niederlande zurückkehren, doch das plötzliche Ableben des ungarischen Königs zwang ihn, sich zunächst um den Erbanspruch der Habsburger in Ungarn zu kümmern. ${ }^{48}$

Neben der noch nicht vollzogenen Hochzeitsnacht sah sich Maximilian weiterem Druck ausgesetzt, da Frankreich in der Bretagne stetig weiter vordrang und Gebiete belagerte (z. B. Nantes Anfang 1491 oder Rennes im Sommer 1491). ${ }^{49}$ Der französische König ging sogar so weit, Anna von Bretagne vor die Wahl zu stellen, entweder ihn zu ehelichen oder ihren "Gatten", den römischen König, mit seinem Hof zu wählen und damit das Herzogtum Bretagne zu verlassen (entgegen regelmäßiger Zahlungen). ${ }^{50}$ „In eine Zwangslage geraten entschied sie sich dafür, [...] in die Hochzeit mit Karl VIII. von Frankreich einzuwilligen." ${ }^{\text {51 }}$ Maximilians Hochzeit durch Stellvertreter hinderte den französischen König nicht daran, dafür zu sorgen, dass diese Vermählung rückgängig gemacht wurde und er selbst besagte Herzogin am 6. Dezember 1491 ehelichte. König Maximilian I. hatte damit nicht nur seine Ehefrau verloren, sondern auch den Ehemann für seine Tochter. ${ }^{52}$

Diese Handlung hatte das Fass zum Überlaufen gebracht - aber nicht nur für MaximiIian. Mit diesem „Brautraub“ hatte sich der französische König auch die Beziehung zu Kaiser Friedrich III. verbaut. ${ }^{53}$ Das lag einerseits an der Hintergehung seines Sohnes, andererseits „empörte den Kaiser die Verstoßung seiner Enkelin, Erzherzogin Margaretes von Österreich-Burgund, die seit mehr als sieben Jahren als Königin von Frankreich tituliert wurde" ${ }^{\prime 54}$.

Dieser Affront gegenüber Friedrich III. erklärt das Ausstellen der untersuchten Quelle, in der zum Reichskrieg gegen Frankreich aufgefordert wird. Wiesflecker spricht allerdings zu diesem fortgeschrittenen Zeitpunkt des Konflikts von einem „letzte[n] Waffenrasseln"55, denn Maximilian wollte endlich heimkehren. Tatsächlich kam es circa einen Monat nach dem Ausstellungsdatum der Quelle zum Waffenstillstand mit Frankreich und im Mai 1493 sogar zum Frieden von Senlis. ${ }^{56}$ Diese enge Abfolge von Kriegsaufruf und Friedensschluss ergibt sich aus Maximilians politischer Strategie, "sich die Entscheidung für Krieg oder Frieden so lange wie möglich offenhalten zu können"57.

Für die Entstehung der Vollmacht können außerdem die gesundheitlichen Umstände des Kaisers im Entstehungsjahr 1493 nicht außer Acht gelassen werden. Das Jahr stellt mit Blick auf die Habsburger und das Heilige Römische Reich einen Abschluss und gleichzeitig einen Umbruch dar. Es ist nämlich das letzte Jahr der achtjährigen Doppelregierung von Kaiser Friedrich III. (Kaiserkrönung 1452) und seinem Sohn König

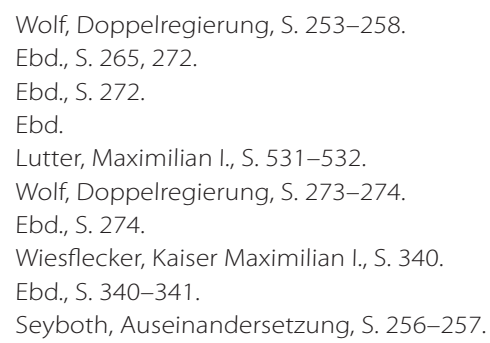


Maximilian I. (Königswahl 1486). ${ }^{58}$ Der 78-jährige Kaiser litt unter "Altersbrand", einer Krankheit, bei der sich Gewebe zurückbildet. Vier Monate nach dem Entstehen der Quelle musste daher das linke Bein des Kaisers, das davon betroffen war, amputiert werden. Kurze Zeit später, im August desselben Jahres, verstarb er schließlich. ${ }^{59}$

Jener Teil der These rund um die Entstehungsgründe hat sich damit bestätigt, den Motiven für das formale Erscheinungsbild der Quelle muss aber noch nachgegangen werden. Im Folgenden stehen daher die Vervielfältigungsgründe im Fokus.

\subsection{Zur Funktion der Kundmachung}

\subsubsection{Zur Nutzung der Druckerzeugnisse unter Maximilian I.}

Die Zeitung als solches sollte zwar erst Jahrzehnte nach Maximilians Tod aufkommen, das stellte für ihn jedoch kein Hindernis dar. Er kommunizierte seine Politik über die von ihm aufgebaute Poststruktur und über Druckerzeugnisse wie Einblattdrucke, was Einheitlichkeit und gleichzeitig umfangreichere Auflagen möglich machte. Im Reich nahm Maximilian I. damit eine führende Position ein..$^{60}$ Seyboth zufolge hatte er "ein ausgesprochenes Gespür für die Relevanz der sogenannten ,öffentlichen Meinung' entwickelt und auch instinktsicher die Einsatz- und Wirkungsmöglichkeiten der noch immer relativ jungen Drucktechnik erkannt"61. Vorteilhaft war dabei auch die Tatsache, dass Informationen schnell und effektiv unter den Fürsten und Untertan*innen verbreitet werden konnten. ${ }^{2}$ Der früheste Einblattdruck, der Maximilian I. als Aussteller anführt, soll im Jahr 1478 entstanden sein und damit 15 Jahre vor der Veröffentlichung der vom Vater ausgestellten Vollmacht. Trotz dieser zeitlichen Diskrepanz gibt es inhaltliche Überschneidungen: In diesem Einblattdruck ging es ebenfalls um militärische Unterstützung für einen Krieg gegen Frankreich (damals noch gegen den Vater Karls VIII.). ${ }^{63}$ Falk Eisermann betont also nicht ohne Grund, dass Maximilian I. den technischen Druck nicht erst als Kaiser für sich genutzt hat, sondern schon zur Zeit der Inkunabeln „Während seiner Mitregentschaft seit 1486 und als Alleinherrscher nach dem Tod Kaiser Friedrichs III.“64 - zum Beispiel bei seiner Wahl zum König 1486.65

Als reine Informationspolitik kann die Nutzung derartiger technischer Hilfsmittel nicht bezeichnet werden. Deutlich kommt das zum Ausdruck, wenn

58 Lutter, Maximilian I., S. 535-536; Alphons Lhotsky, Friedrich III., in: Neue Deutsche Biographie, Bd. 5, Berlin 1961, S. 484-487, https://www.deutsche-biographie.de/sfz56953.html, eingesehen 27.2.2021.

59 Wiesflecker, Kaiser Maximilian I., S. 353.

60 Enno Bünz, Der Kaiser ist tot. Wie das Ableben Maximilians I. 1519 in Kursachsen bekannt gemacht wurde, in: Holger Böning/Hans-Werner Hahn/Alexander Krünes/Uwe Schirmer (Hrsg.), Medien - Kommunikation - Öffentlichkeit. Vom Spätmittelalter bis zur Gegenwart. Festschrift für Werner Greiling zum 65. Geburtstag (Veröffentlichungen der Historischen Kommission für Thüringen, Kleine Reihe 58), Wien-Köln-Weimar 2019, S. 211-234, hier S. $211-$ 213; Müller, Publizistik, S. 95

61 Seyboth, Auseinandersetzung, S. 255

62 Müller, Publizistik, S. 98-99.

63 Falk Eisermann, Buchdruck und politische Kommunikation. Ein neuer Fund zur frühen Publizistik Maximilians I., in: Gutenberg-Jahrbuch 77 (2002), S. 77-83, hier S. 78.

64 Ebd., S. 77.

65 Ebd.; Ursula Bruckner, Coronatio Maximiliani, in: Beiträge zur Inkunabelkunde 8 (1983), Folge 3, S. 94-109. 
„Texte, deren primärer Zweck ein ganz anderer ist [... . von Maximilian und seinen Helfern dafür benutzt werden, um für die Ziele ihrer Politik über einen längeren Zeitraum zu werben, dass also Schrifttum, das primär herrschaftsinternen Zwecken dient, einen die Herrschaft nach außen darstellenden und legitimierenden Nebenzweck erhält." ${ }^{\prime 6}$

Das erklärt auch, wie die in Anlehnung an ihre inhaltlichen und formalen Elemente als Rechtsdokument beschriebene Quelle, die als Rechtsakt die Übertragung der Macht vom Vater auf den Sohn verschriftlicht, eine publizistische Aufbereitung erfahren konnte.

Bei der vorliegenden Quelle handelt es sich, wie bereits erwähnt, nicht um das einzige Dokument, das in das Reich hinausgetragen wurde. Vielmehr soll es eine „mit dem gesamten Repertoire zeitgenössischer Medien geführte propagandistische Auseinandersetzung zwischen Maximilian und Karl VIII." ${ }^{\text {"67 }}$ gegeben haben. In diesem Zusammenhang spielt etwa das von Maximilian I. bewusst erschaffene Narrativ „Brautraub” eine Rolle, das mithilfe von Flugschriften sowie Kulturgütern (z. B. Liedern, Gedichten) verbreitet wurde. In diesen Texten wird die tatsächliche Abreise von Anna von Bretagne nach Frankreich als Entführung mit Gewalt, Leid und aktiver Widersetzung geschildert. Nachträgliche Erklärungen durch die Betroffene selber machten jedoch deutlich, dass diese Verzerrung von Tatsachen Maximilian I. Vorteile verschaffen sollte: Nämlich um den Fürsten des Reiches einen Grund zu geben, um gegen Frankreich in den Krieg zu ziehen und damit die schwerfällige Entscheidungsfindung im Reich zu beschleunigen. In der Literatur ist daher wiederholt der Begriff "Propaganda" zu finden. ${ }^{68} \mathrm{Da}$ diese Vorgehensweise - die Vervielfältigung von Informationen, um die gesellschaftliche Meinung zu beeinflussen ${ }^{69}$ - mehrfach zum Tragen kam, liegt es nahe, dass auch die Vollmacht zum Reichskrieg gegen Frankreich die Reichsfürsten in ihrem Handeln lenken sollte. ${ }^{70}$

„In der Tat war dieser Konflikt primär eine Sache, die nur Maximilian und sein Haus betraf, doch war er sich vollkommen darüber im klaren, daß er allein mit seinen begrenzten Ressourcen gegen den mächtigen französischen König keinesfalls bestehen konnte.".71

\subsubsection{Zur Propaganda Maximilians I. und Friedrichs III.}

Die Vervielfältigung der untersuchten Vollmacht lässt sich in „eine der massivsten publizistisch-propagandistischen Auseinandersetzungen, die Europa bis dahin je erlebt

66 Müller, Publizistik, S. 113.

67 Seyboth, Auseinandersetzung, S. 246.

68 Wiesflecker, Kaiser Maximilian I., S. 335-336; Wolf, Doppelregierung, S. 274-275; Seyboth, Auseinandersetzung, S. 246; Müller, Publizistik, S. 117.

69 Günter Bentele, Propaganda, in: Lexikon Kommunikations- und Medienwissenschaft (Studienbücher zur Kommunikations- und Medienwissenschaft), Wiesbaden 2013, S. 279-280.

70 Seyboth, Auseinandersetzung, S. 256.

71 Ebd., S. 241. 
hatte "72, einordnen. Ein typisches Merkmal der Propaganda-Schriften Maximilians und seines Vaters im Zusammenhang mit Frankreich ist beispielsweise die Fokussierung auf das Reich und die ihm widerfahrene Schmach. Es wurde also weniger auf das Haus Habsburg rekurriert, sondern vielmehr auf die "deutsche Nation", damit die Fürsten bei ihrer Ehre gepackt werden konnten. ${ }^{73}$ Die Propaganda des Vaters und des Sohnes zeichnete sich des Weiteren durch den Topos „Schützen der Christenheit“ aus. Deshalb wurde den Franzosen die christliche Religion zwar nicht abgesprochen, ihre Handlungen wurden aber häufig als den zugrunde liegenden religiösen Wertvorstellungen widersprechend dargestellt. ${ }^{74}$ Der Rückgriff auf diese beiden Strategien lässt sich in der Vollmacht von 1493 in den Worten „den snôden vncristenlichen hanndel so kûnig Karl zû Frannckreich wider sein lieb beganngen hat / vnd seinem pôsen fûrsatz vnd willen / des Er gegen vnns / dem heiligen Reiche vnd gemeiner dewtscher Nation in teglicher ûbung stet $^{\prime \prime 75}$ finden. Mit einem Verweis auf Holleggers Gliederungsversuch zu Maximilians vielschichtigen Propagandahandlungen ${ }^{76}$ soll kritisch hervorgehoben werden, dass mit den vorgestellten Aspekten die Propaganda der beiden Habsburger bei weitem nicht erfasst werden konnte, es bedingt durch den Rahmen einer Proseminararbeit jedoch dabei bleiben muss.

\section{Fazit}

Abschließend sollen die zentralen Inhalte zusammengefasst werden. Die als Inkunabel vorliegende Vollmacht gibt Einblick in die problematische, von Konfrontationen geprägte Beziehung zwischen Maximilian I. und dem französischen König Karl VIII. im 15. Jahrhundert. Mit Friedrich III., Kaiser des Heiligen Römischen Reiches und Vater von Maximilian, als Aussteller dieser Urkunde trifft die Quelle aber auch eine Aussage darüber, welche Position der Kaiser in dieser Auseinandersetzung bezog. Die untersuchte Quelle bringt in Anlehnung an ihre janusköpfige Quellenart - Rechtsdokument in publizistischer Aufbereitung - neben dem Ärger der habsburgischen Herrscher über das Verhalten des französischen Königs auch ihr Vorgehen dagegen zum Ausdruck.

Zur Frage, warum es zur Entstehung der Vollmacht von Kaiser Friedrich III. an König Maximilian im Februar 1493 mit dem Aufruf zur Bestrafung des französischen Königs und deren Vervielfältigung kam, kann nach Analyse der Quelle und intensiver Literaturarbeit Folgendes festgestellt werden: Das jahrelange Ringen zwischen Maximilian I. und Karl VIII. um das Herzogtum Burgund schaukelte sich in wiederkehrenden Auseinandersetzungen - unterbrochen von Friedensverhandlungen und -verträgen - hoch und mündete im sogenannten bretonischen Brautraub. Karl VIII., der seit Jahren mit Maximilians Tochter verlobt war, „stahl“ Maximilians Braut Anna von Bretagne und 
heiratete sie selbst, was schließlich auch den Kaiser erzürnte. In diesen historischen Ereignissen liegt also die Begründung für die Bestrafung.

Die Motive für den Aufruf im Reich - umgesetzt durch die Vervielfältigung - hängen mit der Propaganda Friedrichs III. und der seines Sohnes Maximilian zusammen. Durch die frühe Nutzung von Druckerzeugnissen, die auch bei der vorliegenden Inkunabel zum Tragen kam, konnte rasch, effektiv und flächendeckend die Meinung im Reich beeinflusst werden. Die Doppelregenten mussten die Reichsfürsten von der durch Karl VIII. über das Reich gebrachten Schmach überzeugen, um ihre militärische Unterstützung im Kampf gegen Frankreich sicherzustellen. Ohne diese war ein Sieg über Frankreich nicht umsetzbar. Das vorliegende Schriftstückt reiht sich damit neben anderen im publizistischen Kampf Maximilians gegen Karl VIII. und generell in die Propagandavorgehensweise der Habsburger ein.

Durch die Interpretation innerer und äußerer Merkmale der Quelle konnte somit die aufgestellte These „Die jahrelangen Auseinandersetzungen zwischen Maximilian I. und Karl VIII., aber auch propagandistische Gründe führen zur Ausstellung und Verbreitung der Vollmacht" bestätigt werden.

Für weitere Untersuchungen stellt sich die Frage, ob Karl VIII. eine ähnliche Propagandastrategie verfolgt hat. Es bietet sich auch ein Vergleich bzw. eine Einordnung der vorliegenden Quelle in andere im Rahmen der Auseinandersetzung entstandene Inkunabeln an. So ließe sich der Propagandacharakter der Vollmacht noch deutlicher herausarbeiten sowie der durch sie verursachte verzerrte Blick auf die Situation. Beim Lesen der Vollmacht entsteht der Eindruck von extremer Dringlichkeit des Aufgebotes, was zusätzlich durch das Einschalten des Kaisers verstärkt wird. Wie die Literaturarbeit jedoch ergeben hat, wollte sich Maximilian einfach alle Türen im Kampf gegen Frankreich offen halten. Dies verdeutlicht, dass ein kritischer Blick auf die Quelle von immenser Bedeutung ist.

\section{Quellen}

Vollmacht Kaiser Friedrichs III. an König Maximilian, 1493. TLA, Inkunabel 11, 1493 II 11. Vollmacht Kaiser Friedrichs III. an König Maximilian, 1493. AT-OeStA/HHStA, SB Einblattdrucke 5, https://www.archivinformationssystem.at/detail.aspx?ID=288086, eingesehen 19.2.2021.

\section{Literatur}

Bentele, Günter, Propaganda, in: Lexikon Kommunikations- und Medienwissenschaft (Studienbücher zur Kommunikations- und Medienwissenschaft), Wiesbaden 20132, S. 279-280.

Bruckner, Ursula, Coronatio Maximiliani, in: Beiträge zur Inkunabelkunde 8 (1983), Folge 3, S. 94-109. 
Bünz, Enno, Der Kaiser ist tot. Wie das Ableben Maximilians I. 1519 in Kursachsen bekannt gemacht wurde, in: Holger Böning/Hans-Werner Hahn/Alexander Krünes/Uwe Schirmer (Hrsg.), Medien - Kommunikation - Öffentlichkeit. Vom Spätmittelalter bis zur Gegenwart. Festschrift für Werner Greiling zum 65. Geburtstag (Veröffentlichungen der Historischen Kommission für Thüringen, Kleine Reihe 58), Wien-Köln-Weimar 2019, S. 211-234.

Eisermann, Falk, Buchdruck und politische Kommunikation. Ein neuer Fund zur frühen Publizistik Maximilians I., in: Gutenberg-Jahrbuch 77 (2002), S. 77-83.

Gantert, Klaus, Handschriften, Inkunabeln, Alte Drucke. Informationsressourcen zu historischen Bibliotheksbeständen (Bibliotheks- und Informationspraxis 60), Berlin-Boston 2019.

Heinig, Paul-Joachim (Bearb.), Die Urkunden und Briefe aus dem Stadtarchiv Frankfurt am Main (Regesten Kaiser Friedrichs III. (1440-1493). Nach Archiven und Bibliotheken geordnet 4), Wien-Köln-Graz 1986, http://www.regesta-imperii.de/regesten/ 13-4-0-friedrich-iii/nr/1493-02-11_1_0_13_4_0_10623_1046, eingesehen 19.2.2021.

Hollegger, Manfred, Erwachen und aufsten als ein starcker stryter. Zu Formen und Inhalt der Propaganda Maximilians I., in: Karel Hruza (Hrsg.), Propaganda, Kommunikation und Öffentlichkeit (11.-16. Jahrhundert) (Forschungen zur Geschichte des Mittelalters 6), Wien 2002, S. 223-234.

Lhotsky, Alphons, Friedrich III., in: Neue Deutsche Biographie, Bd. 5, Berlin 1961, S. 484487, https://www.deutsche-biographie.de/sfz56953.html, eingesehen 27.2.2021.

Lutter, Christina, Maximilian I. (1486-1519), in: Bernd Schneidmüller/Stefan Weinfurter (Hrsg.), Die Deutschen Herrscher des Mittelalters. Historische Portraits von Heinrich I. bis Maximilian I. (919-1519), München 2003, S. 518-542.

Müller, Jan-Dirk, Publizistik unter Maximilian I. Zwischen Buchdruck und mündlicher Verkündigung, in: Ute Frevert/Wolfgang Braungart (Hrsg.), Sprachen des Politischen. Medien und Medialität in der Geschichte, Göttingen 2004, S. 95-122.

Seyboth, Reinhard, Die Auseinandersetzung König Maximilians I. mit König Karl VIII. von Frankreich um die Bretagne im Spiegel zeitgenössischer Medien, in: Maximilian Lanzinner/Arno Strohmeyer (Hrsg.), Der Reichstag 1486-1613. Kommunikation - Wahrnehmung - Öffentlichkeiten (Schriftenreihe der Historischen Kommission bei der Bayerischen Akademie der Wissenschaften 73), Göttingen 2006, S. 239-257.

TLA, Quellenedition zu Inkunabel 11, Innsbruck 2019 (unveröffentlichtes Manuskript). Kopie im Besitz der Verfasserin.

Vogtherr, Thomas, Einführung in die Urkundenlehre, Stuttgart $2017^{2}$.

Wiesflecker, Hermann, Kaiser Maximilian I. Das Reich, Österreich und Europa an der Wende zur Neuzeit, Bd. 1: Jugend, burgundisches Erbe und Römisches Königtum bis zur Alleinherrschaft. 1459-1493, Wien-München 1971. 
Wolf, Susanne, Die Doppelregierung Kaiser Friedrichs III. und König Maximilians (14861493) (Forschungen zur Kaiser- und Papstgeschichte des Mittelalters. Beihefte zu J. F. Böhmer, Regesta Imperii 25), Köln-Mainz 2005.

Marina Blum ist Lehramtsstudentin mit den Unterrichtsfächern Geschichte, Sozialkunde, Politische Bildung und Deutsch, Masterstudentin der Germanistik an der Universität Innsbruck sowie studentische Mitarbeiterin am Institut für Zeitgeschichte. marina.blum@student.uibk.ac.at

\section{Zitation dieses Beitrages}

Marina Blum, Eine habsburgische Quelle im Kampf gegen Frankreich: Vollmacht Kaiser Friedrichs III. an König Maximilian, 1493, in: historia.scribere 13 (2021), S. 113-126, [http://historia.scribere.at], eingesehen 22.6.2021 (=aktuelles Datum).

(C) Creative Commons Licences 3.0 Österreich unter Wahrung der Urheberrechte der Autorlnnen. 